\title{
WEIGHTED BOUNDEDNESS OF MULTILINEAR OPERATOR ASSOCIATED TO MULTIPLIER OPERATOR FOR THE EXTREME CASES
}

\author{
JINSONG PAN AND LIJUAN TONG \\ Received 20 December, 2014
}

\begin{abstract}
We prove the weighted boundedness properties for the multilinear operator associated to the multiplier operator for the extreme cases.
\end{abstract}

2010 Mathematics Subject Classification: 42B20; 42B25

Keywords: multilinear operator, multiplier operator, BMO space

\section{INTRODUCTION AND PRELIMINARIES}

As the development of singular integral operators, their commutators and multilinear operators have been well studied (see [1-4, 6,13-15]). Let $T$ be the CalderónZygmund singular integral operator and $b \in B M O\left(R^{n}\right)$, a classical result of Coifman, Rochberg and Weiss (see [5]) stated that the commutator $[b, T](f)=T(b f)-$ $b T(f)$ is bounded on $L^{p}\left(R^{n}\right)$ for $1<p<\infty$. In [7], authors obtain the boundedness properties of the commutators for the extreme values of $p$ are obtained (that is $p=1$ and $p=\infty)$. And note that $[b, T]$ is not bounded for the end point boundedness. The purpose of this paper is to introduce some multilinear operator associated to the multiplier operator and prove the weighted boundedness properties of the multilinear operator for the extreme cases.

First, let us introduce some preliminaries (see $[6,15]$ ). Throughout this paper, $Q$ will denote a cube of $R^{n}$ with sides parallel to the axes. For a locally integrable functions $b$ and a weight function $w$ (that is a non-negative locally integrable function), let $w(Q)=\int_{Q} w(x) d x, w_{Q}=|Q|^{-1} \int_{Q} w(x) d x$, the weighted sharp function of $b$ is defined by

$$
b^{\#}(x)=\sup _{Q \ni x} \frac{1}{w(Q)} \int_{Q}\left|b(y)-b_{Q}\right| w(y) d y .
$$

We say that $b$ belongs to $B M O(w)$ if $b^{\#}$ belongs to $L^{\infty}(w)$ and define $\|b\|_{B M O(w)}=\left\|b^{\#}\right\|_{L^{\infty}(w)}$. If $w=1$, we denote $B M O(w)=B M O\left(R^{n}\right)$. It has 
been known that (see [15])

$$
\left\|b-b_{2^{k}} Q\right\|_{B M O} \leq C k\|b\|_{B M O} .
$$

We also define the central $B M O$ space by $C M O\left(R^{n}\right)$, which is the space of those functions $f \in L_{l o c}\left(R^{n}\right)$ such that

$$
\|f\|_{C M O}=\sup _{r>1}|Q(0, r)|^{-1} \int_{Q}\left|f(x)-f_{Q}\right| d x<\infty .
$$

It is well-known that (see [15])

$$
\|f\|_{C M O} \approx \sup _{r>1} \inf _{c \in C}|Q(0, r)|^{-1} \int_{Q}|f(x)-c| d x .
$$

Definition 1. Let $1<p<\infty$ and $w$ be a non-negative weight functions on $R^{n}$. We shall call $B_{p}(w)$ the space of those functions $f$ on $R^{n}$ such that

$$
\|f\|_{B_{p}(w)}=\sup _{r>1}[w(Q(0, r))]^{-1 / p}\left\|f \chi_{Q(0, r)}\right\|_{L^{p}(w)}<\infty .
$$

The $A_{p}$ weight is defined by (see [6]), for $1<p<\infty$,

$$
\begin{aligned}
A_{p}=\{0<w & \in L_{l o c}^{1}\left(R^{n}\right): \\
& \left.\sup _{Q}\left(\frac{1}{|Q|} \int_{Q} w(x) d x\right)\left(\frac{1}{|Q|} \int_{Q} w(x)^{-1 /(p-1)} d x\right)^{p-1}<\infty\right\}
\end{aligned}
$$

and

$$
A_{1}=\left\{0<w \in L_{l o c}^{1}\left(R^{n}\right): \sup _{Q \ni x} \frac{1}{|Q|} \int_{Q} w(y) d y \leq C w(x), \text { a.e. }\right\} \text {. }
$$

\section{THEOREMS}

In this paper, we will study the multilinear operator as following (see [8]).

A bounded measurable function $k$ defined on $R^{n} \backslash\{0\}$ is called a multiplier. The multiplier operator $T$ associated with $k$ is defined by

$$
T(f)(x)=k(x) \hat{f}(x), \text { for } f \in S\left(R^{n}\right),
$$

where $\hat{f}$ denotes the Fourier transform of $f$ and $S\left(R^{n}\right)$ is the Schwartz test function class. Now, we recall the definition of the class $M(s, l)$. Denote by $|x| \sim t$ the fact that the value of $x$ lies in the annulus $\left\{x \in R^{n}: a t<|x|<b t\right\}$, where $0<a \leq 1<$ $b<\infty$ are values specified in each instance.

Definition 2. Let $l \geq 0$ be a real number and $1 \leq s \leq 2$. we say that the multiplier $k$ satisfies the condition $M(s, l)$, if

$$
\left(\int_{|\xi| \sim R}\left|D^{\alpha} k(\xi)\right|^{s} d \xi\right)^{\frac{1}{s}}<C R^{n / s-|\alpha|}
$$


for all $R>0$ and multi-indices $\alpha$ with $|\alpha| \leq l$, when $l$ is a positive integer, and, in addition, if

$$
\left(\int_{|\xi| \sim R}\left|D^{\alpha} k(\xi)-D^{\alpha} k(\xi-z)\right|^{s} d \xi\right)^{\frac{1}{s}} \leq C\left(\frac{|z|}{R}\right)^{\gamma} R^{\frac{n}{s}-|\alpha|}
$$

for all $|z|<R / 2$ and all multi-indices $\alpha$ with $|\alpha|=[l]$, the integer part of $l$,i.e., $[l]$ is the greatest integer less than or equal to $l$, and $l=[l]+\gamma$ when $l$ is not an integer.

Definition 3. For a real number $\tilde{l} \geq 0$ and $1 \leq \tilde{s}<\infty$, we say that $K$ verifies the condition $\tilde{M}(\tilde{s}, \tilde{l})$, and write $K \in \tilde{M}(\tilde{s}, \tilde{l})$, if

$$
\left(\int_{|x| \sim R}\left|D^{\tilde{\alpha}} K(x)\right|^{\tilde{s}} d x\right)^{\frac{1}{\tilde{s}}} \leq C R^{n / \tilde{s}-n-|\tilde{\alpha}|}, \quad R>0
$$

for all multi-indices $|\tilde{\alpha}| \leq \tilde{l}$ and, in addition, if

$$
\begin{aligned}
& \left(\int_{|x| \sim R}\left|D^{\tilde{\alpha}} K(x)-D^{\tilde{\alpha}} K(x-z)\right|^{\tilde{s}} d x\right)^{\frac{1}{\tilde{s}}} \leq C\left(\frac{|z|}{R}\right)^{v} R^{\frac{n}{\tilde{s}}-n-u}, \quad \text { if } 0<v<1, \\
& \left(\int_{|x| \sim R}\left|D^{\tilde{\alpha}} K(x)-D^{\tilde{\alpha}} K(x-z)\right|^{\tilde{s}} d x\right)^{\frac{1}{\tilde{s}}} \leq C\left(\frac{|z|}{R}\right)\left(\log \frac{R}{|z|}\right) R^{\frac{n}{\tilde{s}}-n-u}, \quad \text { if } \quad v=1,
\end{aligned}
$$

for all $|z|<\frac{R}{2}, R>0$, and all multi-indices $\tilde{\alpha}$ with $|\tilde{\alpha}|=u$, where $u$ denotes the largest integer strictly less than $\tilde{l}$ with $\tilde{l}=u+v$.

Denote $D\left(R^{n}\right)=\left\{\phi \in S\left(R^{n}\right): \operatorname{supp}(\phi)\right.$ is compact $\}$ and $\hat{D}_{0}\left(R^{n}\right)=\left\{\phi \in S\left(R^{n}\right)\right.$ : $\hat{\phi} \in D\left(R^{n}\right)$ and $\hat{\phi}$ vanishes in a neightbourhood of the origin\}. The following boundedness property of $T$ on $L^{p}\left(R^{n}\right)$ is proved by Strömberg and Torkinsky (see [8,12]).

Lemma 1 ([8]). Let $k \in M(s, l), 1 \leq s \leq 2$, and $l>\frac{n}{s}$. Then the associated mapping $T$, defined a priori for $f \in \hat{D}_{0}\left(R^{n}\right), T(f)(x)=(f * K)(x)$, extends to a bounded mapping from $L^{p}\left(R^{n}\right)$ into itself for $1<p<\infty$ and $K(x)=\check{k}(x)$.

Lemma 2 ([8]). Suppose $k \in M(s, l), 1 \leq s \leq 2$. Given $1 \leq \tilde{s}<\infty$, let $r \geq 1$ be such that $\frac{1}{r}=\max \left\{\frac{1}{s}, 1-\frac{1}{\tilde{s}}\right\}$. Then $K \in \tilde{M}(\tilde{s}, \tilde{l})$, where $\tilde{l}=l-\frac{n}{r}$.

Lemma 3 ([8]). Let $1 \leq s<\infty$, suppose that $l$ is a positive real number with $l>n / r, 1 / r=\max \{1 / s, 1-1 / \tilde{s}\}$, and $k \in M(s, l)$. Then there is a positive constant a, such that

$$
\left(\int_{Q_{k}}\left|K(x-z)-K\left(x_{Q}-z\right)\right|^{\tilde{s}} d z\right)^{1 / \tilde{s}} \leq C 2^{-k a}\left(2^{k} h\right)^{-n / \tilde{s}^{\prime}} .
$$

Now we can define the multilinear operator associated to the multiplier operator as following. 
Let $m_{j}$ be the positive integers $(j=1, \cdots, l), m_{1}+\cdots+m_{l}=m$ and $b_{j}$ be the functions on $R^{n}(j=1, \cdots, l)$. Set, for $1 \leq j \leq l$,

$$
R_{m_{j}+1}\left(b_{j} ; x, y\right)=b_{j}(x)-\sum_{|\alpha| \leq m_{j}} \frac{1}{\alpha !} D^{\alpha} b_{j}(y)(x-y)^{\alpha} .
$$

Let $T$ be the multiplier operator. By Lemma $1, T(f)(x)=(K * f)(x)$ for $K(x)=$ $\check{k}(x)$. The multilinear operator associated to $T$ is defined by

$$
T_{b}(f)(x)=\int_{R^{n}} \frac{\prod_{j=1}^{l} R_{m_{j}+1}\left(b_{j} ; x, y\right)}{|x-y|^{m}} K(x-y) f(y) d y .
$$

Note that when $m=0, T_{b}$ is just multilinear commutators of $T$ and $b$ (see [13,14]). It is well-known that multilinear operator, as a non-trivial extension of commutator, is of great interest in harmonic analysis and has been widely studied by many authors (see [1-4]). In this paper, we will study the weighted boundedness properties of the multilinear operator $T_{b}$ for the extreme cases(see [9-11, 16-19]).

We shall prove the following theorems in Section 3.

Theorem 1. Let $T$ be the multiplier operator, $w \in A_{1}$ and $D^{\alpha} b_{j} \in B M O\left(R^{n}\right)$ for all $\alpha$ with $|\alpha|=m_{j}$ and $j=1, \cdots, l$. Then $T_{b}$ is bounded from $L^{\infty}(w)$ to $B M O(w)$.

Theorem 2. Let $T$ be the multiplier operator, $1<p<\infty, w \in A_{1}$ and $D^{\alpha} b_{j} \in$ $B M O\left(R^{n}\right)$ for all $\alpha$ with $|\alpha|=m_{j}$ and $j=1, \cdots, l$. Then $T_{b}$ is bounded from $B_{p}(w)$ to $C M O(w)$.

\section{Proofs OF THEOREMS}

We begin with some preliminaries lemmas.

Lemma 4 ([3]). Let $b$ be a function on $R^{n}$ and $D^{\alpha} b \in L^{q}\left(R^{n}\right)$ for $|\alpha|=m$ and some $q>n$. Then

$$
\left|R_{m}(b ; x, y)\right| \leq C|x-y|^{m} \sum_{|\alpha|=m}\left(\frac{1}{|\tilde{Q}(x, y)|} \int_{\tilde{Q}(x, y)}\left|D^{\alpha} b(z)\right|^{q} d z\right)^{1 / q},
$$

where $\tilde{Q}(x, y)$ is the cube centered at $x$ and having side length $5 \sqrt{n}|x-y|$.

Lemma 5 ([8]). Let $T$ be the multiplier operator. Then $T$ is bounded on $L^{p}\left(R^{n}, w\right)$ for $w \in A_{p}$ with $1<p<\infty$.

Proof of Theorem 1. It is only to prove that there exists a constant $C_{Q}$ such that

$$
\frac{1}{w(Q)} \int_{Q}\left|T_{b}(f)(x)-C_{Q}\right| w(x) d x \leq C\|f\|_{L^{\infty}(w)}
$$

holds for any cube $Q$. Without loss of generality, we may assume $l=2$. Fix a cube $Q=Q\left(x_{0}, d\right)$. Let $\tilde{Q}=5 \sqrt{n} Q$ and $\tilde{b}_{j}(x)=b_{j}(x)-\sum_{|\alpha|=m} \frac{1}{\alpha !}\left(D^{\alpha} b_{j}\right) \tilde{Q}^{x^{\alpha}}$, 
then $R_{m}\left(b_{j} ; x, y\right)=R_{m}\left(\tilde{b}_{j} ; x, y\right)$ and $D^{\alpha} \tilde{b}_{j}=D^{\alpha} b_{j}-\left(D^{\alpha} b_{j}\right) \tilde{Q}$ for $|\alpha|=m_{j}$. We write, for $f_{1}=f \chi_{\tilde{Q}}$ and $f_{2}=f \chi_{R^{n} \backslash \tilde{Q}}$,

$$
\begin{aligned}
& T_{b}(f)(x)=\int_{R^{n}} \frac{\prod_{j=1}^{2} R_{m_{j}+1}\left(\tilde{b}_{j} ; x, y\right)}{|x-y|^{m}} K(x-y) f(y) d y \\
& =\quad \int_{R^{n}} \frac{\prod_{j=1}^{2} R_{m_{j}}\left(\tilde{b}_{j} ; x, y\right)}{|x-y|^{m}} K(x-y) f_{1}(y) d y \\
& -\quad \sum_{\left|\alpha_{1}\right|=m_{1}} \frac{1}{\alpha_{1} !} \int_{R^{n}} \frac{R_{m_{2}}\left(\tilde{b}_{2} ; x, y\right)(x-y)^{\alpha_{1}} D^{\alpha_{1}} \tilde{b}_{1}(y)}{|x-y|^{m}} K(x-y) f_{1}(y) d y \\
& -\quad \sum_{\left|\alpha_{2}\right|=m_{2}} \frac{1}{\alpha_{2} !} \int_{R^{n}} \frac{R_{m_{1}}\left(\tilde{b}_{1} ; x, y\right)(x-y)^{\alpha_{2}} D^{\alpha_{2}} \tilde{b}_{2}(y)}{|x-y|^{m}} K(x-y) f_{1}(y) d y \\
& +\sum_{\substack{\left|\alpha_{1}\right|=m_{1} \\
\left|\alpha_{2}\right|=m_{2}}} \frac{1}{\alpha_{1} ! \alpha_{2} !} \int_{R^{n}} \frac{(x-y)^{\alpha_{1}+\alpha_{2}} D^{\alpha_{1}} \tilde{b}_{1}(y) D^{\alpha_{2}} \tilde{b}_{2}(y)}{|x-y|^{m}} K(x-y) f_{1}(y) d y \\
& +\quad \int_{R^{n}} \frac{\prod_{j=1}^{2} R_{m_{j}+1}\left(\tilde{b}_{j} ; x, y\right)}{|x-y|^{m}} K(x-y) f_{2}(y) d y \\
& =\quad T\left(\frac{\prod_{j=1}^{2} R_{m_{j}}\left(\tilde{b}_{j} ; x, \cdot\right)}{|x-\cdot|^{m}} f_{1}\right) \\
& -\quad T\left(\sum_{\left|\alpha_{1}\right|=m_{1}} \frac{1}{\alpha_{1} !} \frac{R_{m_{2}}\left(\tilde{b}_{2} ; x, \cdot\right)(x-\cdot)^{\alpha_{1}} D^{\alpha_{1}} \tilde{b}_{1}}{|x-\cdot|^{m}} f_{1}\right) \\
& -\quad T\left(\sum_{\left|\alpha_{2}\right|=m_{2}} \frac{1}{\alpha_{2} !} \frac{R_{m_{1}}\left(\tilde{b}_{1} ; x, \cdot\right)(x-\cdot)^{\alpha_{2}} D^{\alpha_{2}} \tilde{b}_{2}}{|x-\cdot|^{m}} f_{1}\right) \\
& +\quad T\left(\sum_{\left|\alpha_{1}\right|=m_{1},\left|\alpha_{2}\right|=m_{2}} \frac{1}{\alpha_{1} ! \alpha_{2} !} \frac{(x-\cdot)^{\alpha_{1}+\alpha_{2}} D^{\alpha_{1}} \tilde{b}_{1} D^{\alpha_{2}} \tilde{b}_{2}}{|x-\cdot|^{m}} f_{1}\right) \\
& +\quad T\left(\frac{\prod_{j=1}^{2} R_{m_{j}+1}\left(\tilde{b}_{j} ; x, \cdot\right)}{|x-\cdot|^{m}} f_{2}\right) \text {, }
\end{aligned}
$$

then

$$
\left|T_{b}(f)(x)-T_{\tilde{b}}\left(f_{2}\right)\left(x_{0}\right)\right| \leq\left|T\left(\frac{\prod_{j=1}^{2} R_{m_{j}}\left(\tilde{b}_{j} ; x, \cdot\right)}{|x-\cdot|^{m}} f_{1}\right)\right|
$$




$$
\begin{aligned}
& +\quad\left|T\left(\sum_{\left|\alpha_{1}\right|=m_{1}} \frac{1}{\alpha_{1} !} \frac{R_{m_{2}}\left(\tilde{b}_{2} ; x, \cdot\right)(x-\cdot)^{\alpha_{1}} D^{\alpha_{1}} \tilde{b}_{1}}{|x-\cdot|^{m}} f_{1}\right)\right| \\
& +\left|T\left(\sum_{\left|\alpha_{2}\right|=m_{2}} \frac{1}{\alpha_{2} !} \frac{R_{m_{1}}\left(\tilde{b}_{1} ; x, \cdot\right)(x-\cdot)^{\alpha_{2}} D^{\alpha_{2}} \tilde{b}_{2}}{|x-\cdot|^{m}} f_{1}\right)\right| \\
& +\quad\left|T\left(\sum_{\left|\alpha_{1}\right|=m_{1},\left|\alpha_{2}\right|=m_{2}} \frac{1}{\alpha_{1} ! \alpha_{2} !} \frac{(x-\cdot)^{\alpha_{1}+\alpha_{2}} D^{\alpha_{1}} \tilde{b}_{1} D^{\alpha_{2}} \tilde{b}_{2}}{|x-\cdot|^{m}} f_{1}\right)\right| \\
& =
\end{aligned}
$$

and

$$
\begin{aligned}
& \frac{1}{w(Q)} \int_{Q}\left|T_{b}(f)(x)-T_{\tilde{b}}\left(f_{2}\right)\left(x_{0}\right)\right| w(x) d x \\
& \leq \frac{1}{w(Q)} \int_{Q} I_{1}(x) w(x) d x+\frac{1}{w(Q)} \int_{Q} I_{2}(x) w(x) d x+\frac{1}{w(Q)} \int_{Q} I_{3}(x) w(x) d x \\
&+\frac{1}{w(Q)} \int_{Q} I_{4}(x) w(x) d x+\frac{1}{w(Q)} \int_{Q} I_{5}(x) w(x) d x \\
& I_{1}+I_{2}+I_{3}+I_{4}+I_{5} .
\end{aligned}
$$

Now, let us estimate $I_{1}, I_{2}, I_{3}, I_{4}$ and $I_{5}$, respectively. First, for $x \in Q$ and $y \in \tilde{Q}$, by Lemma 4, we get

$$
R_{m}\left(\tilde{b}_{j} ; x, y\right) \leq C|x-y|^{m} \sum_{\left|\alpha_{j}\right|=m}\left\|D^{\alpha_{j}} b_{j}\right\|_{B M O},
$$

thus, by the the $L^{p}(w)$-boundedness of $T$ for $1<p<\infty$ Lemma 5 and Hölder's inequality, we obtain

$$
\begin{aligned}
I_{1} & \leq \quad C \prod_{j=1}^{2}\left(\sum_{\left|\alpha_{j}\right|=m_{j}}\left\|D^{\alpha_{j}} b_{j}\right\|_{B M O}\right) \frac{1}{w(Q)} \int_{Q}\left|T\left(f_{1}\right)(x)\right| w(x) d x \\
& \leq C \prod_{j=1}^{2}\left(\sum_{\left|\alpha_{j}\right|=m_{j}}\left\|D^{\alpha_{j}} b_{j}\right\|_{B M O}\right)\left(\frac{1}{w(Q)} \int_{R^{n}}\left|T\left(f_{1}\right)(x)\right|^{p} w(x) d x\right)^{1 / p} \\
& \leq \quad C \prod_{j=1}^{2}\left(\sum_{\left|\alpha_{j}\right|=m_{j}}\left\|D^{\alpha_{j}} b_{j}\right\|_{B M O}\right)\left(\frac{1}{w(Q)} \int_{R^{n}}\left|f_{1}(x)\right|^{p} w(x) d x\right)^{1 / p}
\end{aligned}
$$




$$
\begin{array}{rr}
\leq & C \prod_{j=1}^{2}\left(\sum_{\left|\alpha_{j}\right|=m_{j}}\left\|D^{\alpha_{j}} b_{j}\right\|_{B M O}\right)\left(\frac{w(\tilde{Q})}{w(Q)}\right)^{1 / p}\|f\|_{L^{\infty}(w)} \\
\leq & C \prod_{j=1}^{2}\left(\sum_{\left|\alpha_{j}\right|=m_{j}}\left\|D^{\alpha_{j}} b_{j}\right\|_{B M O}\right)\|f\|_{L^{\infty}(w)} .
\end{array}
$$

For $I_{2}$, since $w \in A_{1}, w$ satisfies the reverse of Hölder' inequality:

$$
\left(\frac{1}{|Q|} \int_{Q} w(x)^{p_{0}} d x\right)^{1 / p_{0}} \leq \frac{C}{|Q|} \int_{Q} w(x) d x
$$

for all cube $Q$ and some $1<p_{0}<\infty$ ( see [6]), thus, by the $L^{p}$-boundedness of $T$ for $p>1$, we get

$$
\begin{array}{rlrl}
I_{2} & \leq & C \sum_{\left|\alpha_{2}\right|=m_{2}}\left\|D^{\alpha_{2}} b_{2}\right\|_{B M O} \sum_{\left|\alpha_{1}\right|=m_{1}} \frac{1}{w(Q)} \int_{Q}\left|T\left(D^{\alpha_{1}} \tilde{b}_{1} f_{1}\right)(x)\right| w(x) d x \\
& \leq \quad C \sum_{\left|\alpha_{2}\right|=m_{2}}\left\|D^{\alpha_{2}} b_{2}\right\|_{B M O} \sum_{\left|\alpha_{1}\right|=m_{1}}\left(\frac{1}{w(Q)} \int_{R^{n}}\left|T\left(D^{\alpha_{1}} \tilde{b}_{1} f_{1}\right)(x)\right|^{p} w(x) d x\right)^{1 / p} \\
& \leq \quad C \sum_{\left|\alpha_{2}\right|=m_{2}}\left\|D^{\alpha_{2}} b_{2}\right\|_{B M O} \sum_{\left|\alpha_{1}\right|=m_{1}}\left(\frac{1}{w(Q)} \int_{R^{n}}\left|D^{\alpha_{1}} \tilde{b}_{1}(x) f_{1}(x)\right|^{p} w(x) d x\right)^{1 / p} \\
& \quad C \sum_{\left|\alpha_{2}\right|=m_{2}}\left\|D^{\alpha_{2}} b_{2}\right\|_{B M O} \sum_{\left|\alpha_{1}\right|=m_{1}}\left(\frac{1}{|Q|} \int_{\tilde{Q}}\left|D^{\alpha_{1}} b_{1}(x)-\left(D^{\alpha_{1}} b_{1}\right)_{\tilde{Q}}\right|^{p p_{0}^{\prime}} d x\right)^{1 / p p_{0}^{\prime}} \\
& \leq \quad C \prod_{j=1}^{2}\left(\sum_{\left|\alpha_{j}\right|=m_{j}}\left\|D^{\alpha_{j}} b_{j}\right\|_{B M O}\right) w(Q)^{-1 / p}|Q|^{1 / p}\left(\frac{1}{|\tilde{Q}|} \int_{\tilde{Q}} w(x)^{p_{0}} d x\right)^{1 / p p_{0}}\|f\|_{L^{\infty}(w)}\left(\frac{1}{|\tilde{Q}|} \int_{\tilde{Q}} w(x) d x\right)^{1 / p}\|f\|_{L^{\infty}(w)} \\
& \leq \quad C \prod_{j=1}^{2}\left(\sum_{\left|\alpha_{j}\right|=m_{j}}\left\|D^{\alpha_{j}} b_{j}\right\|_{B M O}\right)\|f\|_{L^{\infty}(w) .}
\end{array}
$$

For $I_{3}$, similar to the proof of $I_{2}$, we get

$$
I_{3} \leq C \prod_{j=1}^{2}\left(\sum_{\left|\alpha_{j}\right|=m_{j}}\left\|D^{\alpha_{j}} b_{j}\right\|_{B M O}\right)\|f\|_{L^{\infty}(w)} .
$$

Similarly, for $I_{4}$, choose $1<r_{1}, r_{2}<\infty$ such that $1 / r_{1}+1 / r_{2}+1 / p_{0}=1$, we obtain, by Hölder'inequality and reverse of Hölder's inequality,

$$
I_{4} \leq \quad C \sum_{\left|\alpha_{1}\right|=m_{1},\left|\alpha_{2}\right|=m_{2}} \frac{1}{w(Q)} \int_{Q}\left|T\left(D^{\alpha_{1}} \tilde{b}_{1} D^{\alpha_{2}} \tilde{b}_{2} f_{1}\right)(x)\right| w(x) d x
$$




$$
\begin{aligned}
& \leq \quad C \sum_{\left|\alpha_{1}\right|=m_{1},\left|\alpha_{2}\right|=m_{2}}\left(\frac{1}{w(Q)} \int_{R^{n}}\left|T\left(D^{\alpha_{1}} \tilde{b}_{1} D^{\alpha_{2}} \tilde{b}_{2} f_{1}\right)(x)\right|^{p} w(x) d x\right)^{1 / p} \\
& \leq \quad C \sum_{\substack{\left|\alpha_{1}\right|=m_{1},\left|\alpha_{2}\right|=m_{2} \\
\leq}} w(Q)^{-1 / p}\left(\int_{R^{n}}\left|D^{\alpha_{1}} \tilde{b}_{1}(x) D^{\alpha_{2}} \tilde{b}_{2}(x) f_{1}(x)\right|^{p} w(x) d x\right)^{1 / p} \\
& \times \quad C \sum_{\substack{\left|\alpha_{1}\right|=m_{1} \\
\left|\alpha_{2}\right|=m_{2}}}\left(\frac{1}{|\tilde{Q}|} \int_{\tilde{Q}}\left|D^{\alpha_{1}} \tilde{b}_{1}(x)\right|^{p r_{1}} d x\right)^{1 / p r_{1}}\left(\frac{1}{|\tilde{Q}|} \int_{\tilde{Q}}\left|D^{\alpha_{2}} \tilde{b}_{2}(x)\right|^{p r_{2}} d x\right)^{1 / p r_{2}} \\
& \leq \quad w(Q)^{-1 / p}|Q|^{1 / p}\left(\frac{1}{|\tilde{Q}|} \int_{\tilde{Q}} w(x)^{p_{0}} d x\right)^{1 / p p_{0}}\|f\|_{L^{\infty}(w)} \\
& \leq \prod_{j=1}^{2}\left(\sum_{\left|\alpha_{j}\right|=m_{j}}\left\|D^{\alpha_{j}} b_{j}\right\|_{B M O}\right)\|f\|_{L^{\infty}(w) .} .
\end{aligned}
$$

For $I_{5}$, we write, for $x \in Q$,

$$
\begin{aligned}
& =\quad \int_{R^{n}}\left(K(x-y)-K\left(x_{0}-y\right)\right) \frac{1}{|x-y|^{m}} \prod_{j=1}^{2} R_{m_{j}}\left(\tilde{b}_{j} ; x, y\right) f_{2}(y) d y \\
& +\quad \int_{R^{n}}\left(\frac{1}{|x-y|^{m}}-\frac{1}{\left|x_{0}-y\right|^{m}}\right) K\left(x_{0}-y\right) \prod_{j=1}^{2} R_{m_{j}}\left(\tilde{b}_{j} ; x, y\right) f_{2}(y) d y \\
& +\quad \int_{R^{n}}\left(R_{m_{1}}\left(\tilde{b}_{1} ; x, y\right)-R_{m_{1}}\left(\tilde{b}_{1} ; x_{0}, y\right)\right) \frac{R_{m_{2}}\left(\tilde{b}_{2} ; x, y\right)}{\left|x_{0}-y\right|^{m}} K\left(x_{0}-y\right) f_{2}(y) d y \\
& +\quad \int_{R^{n}}\left(R_{m_{2}}\left(\tilde{b}_{2} ; x, y\right)-R_{m_{2}}\left(\tilde{b}_{2} ; x_{0}, y\right)\right) \frac{R_{m_{1}}\left(\tilde{b}_{1} ; x_{0}, y\right)}{\left|x_{0}-y\right|^{m}} K\left(x_{0}-y\right) f_{2}(y) d y \\
& -\quad \sum_{\alpha_{1} \mid=m_{1}} \frac{1}{\alpha_{1} !} \int_{R^{n}}\left[\frac{R_{m_{2}}\left(\tilde{b}_{2} ; x, y\right)(x-y)^{\alpha_{1}}}{|x-y|^{m}} K(x-y)\right. \\
& \left.-\quad \frac{R_{m_{2}}\left(\tilde{b}_{2} ; x_{0}, y\right)\left(x_{0}-y\right)^{\alpha_{1}}}{\left|x_{0}-y\right|^{m}} K\left(x_{0}-y\right)\right] D^{\alpha_{1}} \tilde{b}_{1}(y) f_{2}(y) d y \\
& \sum_{\left|\alpha_{2}\right|=m_{2}} \frac{1}{\alpha_{2} !} \int_{R^{n}}\left[\frac{R_{m_{1}}\left(\tilde{b}_{1} ; x, y\right)(x-y)^{\alpha_{2}}}{|x-y|^{m}} K(x-y)\right. \\
& \left.\frac{R_{m_{1}}\left(\tilde{b}_{1} ; x_{0}, y\right)\left(x_{0}-y\right)^{\alpha_{2}}}{\left|x_{0}-y\right|^{m}} K\left(x_{0}-y\right)\right] D^{\alpha_{2}} \tilde{b}_{2}(y) f_{2}(y) d y
\end{aligned}
$$




$$
\begin{aligned}
& +\quad \sum_{\left|\alpha_{1}\right|=m_{1},\left|\alpha_{2}\right|=m_{2}} \frac{1}{\alpha_{1} ! \alpha_{2} !} \int_{R^{n}}\left[\frac{(x-y)^{\alpha_{1}+\alpha_{2}}}{|x-y|^{m}} K(x-y)\right. \\
& - \\
& \left.\frac{\left(x_{0}-y\right)^{\alpha_{1}+\alpha_{2}}}{\left|x_{0}-y\right|^{m}} K\left(x_{0}-y\right)\right] D^{\alpha_{1}} \tilde{b}_{1}(y) D^{\alpha_{2}} \tilde{b}_{2}(y) f_{2}(y) d y \\
& =\quad I_{5}^{(1)}(x)+I_{5}^{(2)}(x)+I_{5}^{(3)}(x)+I_{5}^{(4)}(x)+I_{5}^{(5)}(x)+I_{5}^{(6)}(x)+I_{5}^{(7)}(x) .
\end{aligned}
$$

By Lemma 4 and the following inequality (see [15])

$$
\left|b_{Q_{1}}-b_{Q_{2}}\right| \leq C \log \left(\left|Q_{2}\right| /\left|Q_{1}\right|\right)|| b \|_{B M O} \text { for } Q_{1} \subset Q_{2},
$$

we know that, for $x \in Q$ and $y \in 2^{k+1} \tilde{Q} \backslash 2^{k} \tilde{Q}$,

$$
\begin{aligned}
\left|R_{m_{j}}\left(\tilde{b}_{j} ; x, y\right)\right| & \leq C|x-y|^{m_{j}} \sum_{|\alpha|=m_{j}}\left(|| D^{\alpha} b_{j} \|_{B M O}+\left|\left(D^{\alpha} b_{j}\right) \tilde{Q}(x, y)-\left(D^{\alpha} b_{j}\right)_{\tilde{Q}}\right|\right) \\
& \leq \\
& C k|x-y|^{m_{j}} \sum_{|\alpha|=m_{j}}\left\|D^{\alpha} b_{j}\right\|_{B M O} .
\end{aligned}
$$

Note that $|x-y| \sim\left|x_{0}-y\right|$ for $x \in Q$ and $y \in R^{n} \backslash \tilde{Q}$, we obtain, by the conditions on $K$ and Lemma 3,

$$
\begin{aligned}
& \left|I_{5}^{(1)}(x)\right| \\
& \leq \quad \sum_{k=0}^{\infty} \int_{2^{k+1} \tilde{Q} \backslash 2^{k} \tilde{Q}}\left|K(x-y)-K\left(x_{0}-y\right)\right|\left|x_{0}-y\right|^{-m} \prod_{j=1}^{2}\left|R_{m_{j}}\left(\tilde{b}_{j} ; x, y\right)\right||f(y)| d y \\
& \leq C \prod_{j=1}^{2}\left(\sum_{|\alpha|=m_{j}}\left\|D^{\alpha} b_{j}\right\|_{B M O}\right) \sum_{k=0}^{\infty} \int_{2^{k+1} \tilde{Q} \backslash 2^{k} \tilde{Q}} k^{2}\left|K(x-y)-K\left(x_{0}-y\right) \| f(y)\right| d y \\
& \leq \quad C \prod_{j=1}^{2}\left(\sum_{|\alpha|=m_{j}}\left\|D^{\alpha} b_{j}\right\|_{B M O}\right) \sum_{k=0}^{\infty} k^{2}\left(\int_{2^{k+1} \tilde{Q}}|f(y)|^{s} d y\right)^{1 / s} \\
& \times \quad\left(\int_{2^{k+1} \tilde{Q} \backslash 2^{k} \tilde{Q}}\left|K(x-y)-K\left(x_{0}-y\right)\right|^{s^{\prime}} d y\right)^{1 / s^{\prime}} \\
& \leq \quad C \prod_{j=1}^{2}\left(\sum_{|\alpha|=m_{j}}\left\|D^{\alpha} b_{j}\right\|_{B M O}\right) \sum_{k=1}^{\infty} k^{2} 2^{-k a}\|f\|_{L^{\infty}(w)} \\
& \leq \quad C \prod_{j=1}^{2}\left(\sum_{|\alpha|=m_{j}}\left\|D^{\alpha} b_{j}\right\|_{B M O}\right)\|f\|_{L^{\infty}(w)} .
\end{aligned}
$$

For $I_{5}^{(2)}(x)$, by Lemma 2, we obtain

$$
\left|I_{5}^{(2)}(x)\right|
$$




$$
\begin{array}{r}
\leq \quad C \prod_{j=1}^{2}\left(\sum_{|\alpha|=m_{j}}\left\|D^{\alpha} b_{j}\right\|_{B M O}\right) \sum_{k=0}^{\infty} \int_{2^{k+1} \tilde{Q} \backslash 2^{k} \tilde{Q}} k^{2} \frac{\left|x-x_{0}\right|}{\left|x_{0}-y\right|}\left|K\left(x_{0}-y\right) \| f(y)\right| d y \\
\leq \quad C \prod_{j=1}^{2}\left(\sum_{|\alpha|=m_{j}}\left\|D^{\alpha} b_{j}\right\|_{B M O}\right) \sum_{k=0}^{\infty} k^{2} 2^{-k}\left(\int_{2^{k+1} \tilde{Q} \backslash 2^{k} \tilde{Q}}\left|K\left(x_{0}-y\right)\right|^{s^{\prime}} d y\right)^{1 / s^{\prime}} \\
\times\left(\int_{2^{k+1} \tilde{Q}}|f(y)|^{s} d y\right)^{1 / s} \\
\leq \quad C \prod_{j=1}^{2}\left(\sum_{|\alpha|=m_{j}}\left\|D^{\alpha} b_{j}\right\|_{B M O}\right) \sum_{k=0}^{\infty} k^{2} 2^{-k}|| f \|_{L^{\infty}(w)} \\
\leq \quad C \prod_{j=1}^{2}\left(\sum_{|\alpha|=m_{j}}\left\|D^{\alpha} b_{j}\right\|_{B M O}\right)\|f\|_{L^{\infty}(w) .}
\end{array}
$$

For $I_{5}^{(3)}(x)$, by the formula (see [3]):

$$
R_{m_{j}}\left(\tilde{b}_{j} ; x, y\right)-R_{m_{j}}\left(\tilde{b}_{j} ; x_{0}, y\right)=\sum_{|\beta|<m} \frac{1}{\beta !} R_{m-|\beta|}\left(D^{\beta} \tilde{b}_{j} ; x, x_{0}\right)(x-y)^{\beta}
$$

and Lemma 4, we have

$$
\begin{gathered}
\left|R_{m_{j}}\left(\tilde{b}_{j} ; x, y\right)-R_{m_{j}}\left(\tilde{b}_{j} ; x_{0}, y\right)\right| \\
\leq C \sum_{|\beta|<m_{j}} \sum_{|\alpha|=m_{j}}\left|x-x_{0}\right|^{m_{j}-|\beta|}|x-y|^{|\beta|}|| D^{\alpha} b_{j} \|_{B M O},
\end{gathered}
$$

thus, by Lemma 2, we obtain

$$
\begin{aligned}
& \leq \quad C \prod_{j=1}^{2}\left(\sum_{|\alpha|=m_{j}}\left\|D^{\alpha} b_{j}\right\|_{B M O}\right) \sum_{k=0}^{\infty} \int_{2^{k+1} \tilde{Q} \backslash 2^{k} \tilde{Q}} k^{2} \frac{\left|x-x_{0}\right|}{\left|x_{0}-y\right|}\left|K\left(x_{0}-y\right) \| f(y)\right| d y \\
& \leq \quad C \prod_{j=1}^{2}\left(\sum_{|\alpha|=m_{j}}\left\|D^{\alpha} b_{j}\right\|_{B M O}\right) \sum_{k=0}^{\infty} k^{2} 2^{-k}\left(\int_{2^{k+1} \tilde{Q} \backslash 2^{k} \tilde{Q}}\left|K\left(x_{0}-y\right)\right|^{s^{\prime}} d y\right)^{1 / s^{\prime}} \\
& \leq \quad \times\left(\int_{2^{k+1} \tilde{Q}}|f(y)|^{s} d y\right)^{1 / s} \\
& \leq \prod_{j=1}^{2}\left(\sum_{|\alpha|=m_{j}}\left\|D^{\alpha} b_{j} \mid\right\|_{B M O}\right) \sum_{k=0}^{\infty} k^{2} 2^{-k}\left(2^{k} d\right)^{-n / s}\left(2^{k} d\right)^{n / s}|| f \|_{L^{\infty}(w)} \\
& \leq \quad C \prod_{j=1}^{2}\left(\sum_{|\alpha|=m_{j}}\left\|D^{\alpha} b_{j}\right\|_{B M O}\right)\|f\|_{L^{\infty}(w) .}
\end{aligned}
$$


Similarly,

$$
\left|I_{5}^{(4)}(x)\right| \leq C \prod_{j=1}^{2}\left(\sum_{\left|\alpha_{j}\right|=m_{j}}\left\|D^{\alpha_{j}} b_{j}\right\|_{B M O}\right)\|f\|_{L^{\infty}(w)} .
$$

For $I_{5}^{(5)}(x)$, similar to the proofs of $I_{5}^{(1)}(x), I_{5}^{(2)}(x), I_{5}^{(3)}(x)$ and $I_{4}$, we get, for $1<s_{1}, s_{2}<\infty$ with $1 / s_{1}+1 / s_{2}+1 / s=1$,

$$
\begin{aligned}
& \left|I_{5}^{(5)}(x)\right| \leq \quad C \sum_{\left|\alpha_{1}\right|=m_{1}} \int_{(\tilde{Q})^{c}} \frac{\left|(x-y)^{\alpha_{1}}\right|}{|x-y|^{m}}\left|R_{m_{2}}\left(\tilde{b}_{2} ; x, y\right)-R_{m_{2}}\left(\tilde{b}_{2} ; x_{0}, y\right)\right| \\
& \left|K(x-y) D^{\alpha_{1}} \tilde{b}_{1}(y)\right||f(y)| d y \\
& +\quad C \sum_{\left|\alpha_{1}\right|=m_{1}} \int_{(\tilde{Q})^{c}}\left|\frac{(x-y)^{\alpha_{1}}}{|x-y|^{m}}-\frac{\left(x_{0}-y\right)^{\alpha_{1}}}{\left|x_{0}-y\right|^{m}}\right| \\
& \times \quad\left|R_{m_{2}}\left(\tilde{b}_{2} ; x_{0}, y\right) K(x-y) D^{\alpha_{1}} \tilde{b}_{1}(y)\right||f(y)| d y \\
& +\quad C \sum_{\left|\alpha_{1}\right|=m_{1}} \int_{(\tilde{Q})^{c}} \frac{\left|R_{m_{2}}\left(\tilde{b}_{2} ; x_{0}, y\right)\right|\left|\left(x_{0}-y\right)^{\alpha_{1}}\right|}{\left|x_{0}-y\right|^{m}} \\
& \times \quad\left|K(x-y)-K\left(x_{0}-y\right)\right|\left|D^{\alpha_{1}} \tilde{b}_{1}(y)\right||f(y)| d y \\
& \leq C \sum_{|\alpha|=m_{2}}\left\|D^{\alpha} b_{2}\right\|_{B M O} \sum_{\left|\alpha_{1}\right|=m_{1}} \sum_{k=0}^{\infty} k 2^{-k}\left(\int_{2^{k+1} \tilde{Q} \backslash 2^{k} \tilde{Q}}|K(x-y)|^{s_{1}} d y\right)^{1 / s_{1}} \\
& \times \quad\left(\int_{2^{k+1} \tilde{Q}}\left|D^{\alpha_{1}} \tilde{b}_{1}(y)\right|^{s_{2}} d y\right)^{1 / s_{2}}\left(\int_{2^{k+1} \tilde{Q}}|f(y)|^{s} d y\right)^{1 / s} \\
& +\quad C \sum_{|\alpha|=m_{2}}\left\|D^{\alpha} b_{2}\right\|_{B M O} \\
& \times \quad \sum_{\left|\alpha_{1}\right|=m_{1}} \sum_{k=0}^{\infty} k\left(\int_{2^{k+1} \tilde{Q} \backslash 2^{k} \tilde{Q}}\left|K(x-y)-K\left(x_{0}-y\right)\right|^{s_{1}} d y\right)^{1 / s_{1}} \\
& \times \quad\left(\int_{2^{k+1} \tilde{Q}}\left|D^{\alpha_{1}} \tilde{b}_{1}(y)\right|^{s_{2}} d y\right)^{1 / s_{2}}\left(\int_{2^{k+1} \tilde{Q}}|f(y)|^{s} d y\right)^{1 / s} \\
& \leq \quad C \prod_{j=1}^{2}\left(\sum_{|\alpha|=m_{j}}\left\|D^{\alpha} b_{j}\right\|_{B M O}\right) \sum_{k=1}^{\infty} k^{2}\left(2^{-k}+2^{-k a}\right)\|f\|_{L^{\infty}(w)} \\
& \leq \quad C \prod_{j=1}^{2}\left(\sum_{|\alpha|=m_{j}}\left\|D^{\alpha} b_{j}\right\|_{B M O}\right)\|f\|_{L^{\infty}(w)} .
\end{aligned}
$$


Similarly,

$$
\left|I_{5}^{(6)}(x)\right| \leq C \prod_{j=1}^{2}\left(\sum_{\left|\alpha_{j}\right|=m_{j}}\left\|D^{\alpha_{j}} b_{j}\right\|_{B M O}\right)\|f\|_{L^{\infty}(w)} .
$$

For $I_{5}^{(7)}(x)$, similar to the proofs of $I_{5}^{(5)}(x)$ and $I_{4}$, we get, for $1<s_{1}, s_{2}, s_{3}<\infty$ with $1 / s_{1}+1 / s_{2}+1 / s_{3}+1 / s=1$,

$$
\begin{aligned}
& \left|I_{5}^{(7)}(x)\right| \leq \\
& C \sum_{\left|\alpha_{1}\right|=m_{1},\left|\alpha_{2}\right|=m_{2}} \int_{(\tilde{Q})^{c}}\left|\frac{(x-y)^{\alpha_{1}+\alpha_{2}}}{|x-y|^{m}}-\frac{\left(x_{0}-y\right)^{\alpha_{1}+\alpha_{2}}}{\left|x_{0}-y\right|^{m}}\right| \\
& \left|K(x-y) D^{\alpha_{1}} \tilde{b}_{1}(y) D^{\alpha_{2}} \tilde{b}_{2}(y)\right||f(y)| d y \\
& \times \\
& +\quad C \sum_{\left|\alpha_{1}\right|=m_{1},\left|\alpha_{2}\right|=m_{2}} \int_{(\tilde{Q})^{c}} \frac{\mid\left(x_{0}-y\right)^{\alpha_{1}+\alpha_{2} \mid}}{\left|x_{0}-y\right|^{m}} \\
& \times \quad\left|K(x-y)-K\left(x_{0}-y\right)\right|\left|D^{\alpha_{1}} \tilde{b}_{1}(y) D^{\alpha_{2}} \tilde{b}_{2}(y) \| f(y)\right| d y \\
& \leq \quad C \sum_{\left|\alpha_{1}\right|=m_{1},|\alpha|=m_{2}} \sum_{k=0}^{\infty} 2^{-k}\left(\int_{2^{k+1} \tilde{Q} \backslash 2^{k} \tilde{Q}}|K(x-y)|^{s_{1}} d y\right)^{1 / s_{1}}\left(\int_{2^{k+1} \tilde{Q}}|f(y)|^{s} d y\right)^{1 / s} \\
& \times \quad\left(\int_{2^{k+1} \tilde{Q}}\left|D^{\alpha_{1}} \tilde{b}_{1}(y)\right|^{s_{2}} d y\right)^{1 / s_{2}}\left(\int_{2^{k+1} \tilde{Q}}\left|D^{\alpha_{2}} \tilde{b}_{2}(y)\right|^{s_{3}} d y\right)^{1 / s_{3}} \\
& +\quad C \sum_{\left|\alpha_{1}\right|=m_{1},|\alpha|=m_{2}} \sum_{k=0}^{\infty}\left(\int_{2^{k+1} \tilde{Q} \backslash 2^{k} \tilde{Q}}\left|K(x-y)-K\left(x_{0}-y\right)\right|^{s_{1}} d y\right)^{1 / s_{1}} \\
& \times \quad\left(\int_{2^{k+1} \tilde{Q}}\left|D^{\alpha_{1}} \tilde{b}_{1}(y)\right|^{s_{2}} d y\right)^{1 / s_{2}}\left(\int_{2^{k+1} \tilde{Q}}\left|D^{\alpha_{2}} \tilde{b}_{2}(y)\right|^{s_{3}} d y\right)^{1 / s_{3}}\left(\int_{2^{k+1} \tilde{Q}}|f(y)|^{s} d y\right)^{1 / s} \\
& \leq \quad C \prod_{j=1}^{2}\left(\sum_{|\alpha|=m_{j}}\left\|D^{\alpha} b_{j}\right\|_{B M O}\right) \sum_{k=1}^{\infty} k^{2}\left(2^{-k}+2^{-k a}\right)\|f\|_{L^{\infty}(w)} \\
& \leq \quad C \prod_{j=1}^{2}\left(\sum_{|\alpha|=m_{j}}\left\|D^{\alpha} b_{j}\right\|_{B M O}\right)\|f\|_{L^{\infty}(w)} .
\end{aligned}
$$

Thus

$$
I_{5} \leq C \prod_{j=1}^{2}\left(\sum_{\left|\alpha_{j}\right|=m_{j}}\left\|D^{\alpha_{j}} b_{j}\right\|_{B M O}\right)\|f\|_{L^{\infty}(w)} .
$$

This completes the proof of Theorem 1 .

Proof of Theorem 2. It is only to prove that there exists a constant $C_{Q}$ such that

$$
\frac{1}{w(Q)} \int_{Q}\left|T_{b}(f)(x)-C_{Q}\right| w(x) d x \leq C\|f\|_{B_{p}(w)}
$$

holds for any cube $Q=Q(0, d)$ with $d>1$. Without loss of generality, we may assume $l=2$. Fix a cube $Q=Q(0, d)$ with $d>1$. Let $\tilde{Q}=5 \sqrt{n} Q$ and $\tilde{b}_{j}(x)=$ $b_{j}(x)-\sum_{|\alpha|=m_{j}} \frac{1}{\alpha !}\left(D^{\alpha} b_{j}\right) \tilde{Q}^{x^{\alpha}}$, then $R_{m_{j}}\left(b_{j} ; x, y\right)=R_{m_{j}}\left(\tilde{b}_{j} ; x, y\right)$ and $D^{\alpha} \tilde{b}_{j}=D^{\alpha} b_{j}-$ 
$\left(D^{\alpha} b_{j}\right)_{\tilde{Q}}$ for $|\alpha|=m_{j}$. Similar to the proof of Theorem 1, we write, for $f_{1}=f \chi_{\tilde{Q}}$ and $f_{2}=f \chi_{R^{n} \backslash \tilde{Q}}$,

$$
\begin{aligned}
& \frac{1}{w(Q)} \int_{Q}\left|T_{b}(f)(x)-T_{\tilde{b}}\left(f_{2}\right)(0)\right| w(x) d x \\
\leq & \frac{1}{w(Q)} \int_{Q}\left|T\left(\frac{\prod_{j=1}^{2} R_{m_{j}}\left(\tilde{b}_{j} ; x, \cdot\right)}{|x-\cdot|^{m}} f_{1}\right)\right| w(x) d x \\
+ & \frac{1}{w(Q)} \int_{Q}\left|T\left(\sum_{\left|\alpha_{1}\right|=m_{1}} \frac{1}{\alpha_{1} !} \frac{R_{m_{2}}\left(\tilde{b}_{2} ; x, \cdot\right)(x-\cdot)^{\alpha_{1}} D^{\alpha_{1}} \tilde{b}_{1}}{|x-\cdot|^{m}} f_{1}\right)\right| w(x) d x \\
+ & \frac{1}{w(Q)} \int_{Q}\left|T\left(\sum_{\left|\alpha_{2}\right|=m_{2}} \frac{1}{\alpha_{2} !} \frac{R_{m_{1}}\left(\tilde{b}_{1} ; x, \cdot\right)(x-\cdot)^{\alpha_{2}} D^{\alpha_{2}} \tilde{b}_{2}}{|x-\cdot|^{m}} f_{1}\right)\right| w(x) d x \\
+ & \frac{1}{w(Q)} \int_{Q}\left|T\left(\sum_{\left|\alpha_{1}\right|=m_{1},\left|\alpha_{2}\right|=m_{2}} \frac{1}{\alpha_{1} ! \alpha_{2} !} \frac{(x-\cdot)^{\alpha_{1}+\alpha_{2}} D^{\alpha_{1}} \tilde{b}_{1} D^{\alpha_{2}} \tilde{b}_{2}}{|x-\cdot|^{m}} f_{1}\right)\right| w(x) d x \\
+ & \frac{1}{w(Q)} \int_{Q}\left|T_{\tilde{b}}\left(f_{2}\right)(x)-T_{\tilde{b}}\left(f_{2}\right)(0)\right| w(x) d x \\
= & L_{1}+L_{2}+L_{3}+L_{4}+L_{5} .
\end{aligned}
$$

Similar to the proof of Theorem 1, we get

$$
\begin{array}{rlrl}
L_{1} & \leq C \prod_{j=1}^{2}\left(\sum_{\left|\alpha_{j}\right|=m_{j}}\left\|D^{\alpha_{j}} b_{j}\right\|_{B M O}\right)\left(\frac{1}{w(Q)} \int_{R^{n}}\left|T\left(f_{1}\right)(x)\right|^{p} w(x) d x\right)^{1 / p} \\
& \leq \\
& \leq \prod_{j=1}^{2}\left(\sum_{\left|\alpha_{j}\right|=m_{j}}\left\|D^{\alpha_{j}} b_{j}\right\|_{B M O}\right) w(\tilde{Q})^{-1 / p}\left\|f \chi_{\tilde{Q}}\right\|_{L^{p}(w)} \\
C \prod_{j=1}^{2}\left(\sum_{\left|\alpha_{j}\right|=m_{j}}\left\|D^{\alpha_{j}} b_{j}\right\|_{B M O}\right)\|f\|_{B_{p}(w)} .
\end{array}
$$

For $L_{2}$, taking $r, s, t>1$ such that $r<p, t=p p_{0} /(p-r)$ and $1 / s+1 /(p / r)+$ $1 / t=1$, then, by the reverse of Hölder's inequality,

$$
\begin{array}{rlrl}
L_{2} & \leq & \quad C \sum_{\left|\alpha_{2}\right|=m_{2}}\left\|D^{\alpha_{2}} b_{2}\right\|_{B M O} \sum_{\left|\alpha_{1}\right|=m_{1}}\left(\frac{1}{w(Q)} \int_{R^{n}}\left|T\left(D^{\alpha_{1}} \tilde{b}_{1} f_{1}\right)(x)\right|^{r} w(x) d x\right)^{1 / r} \\
& \leq \quad C \sum_{\left|\alpha_{2}\right|=m_{2}}\left\|D^{\alpha_{2}} b_{2}\right\|_{B M O} w(Q)^{-1 / r} \sum_{\left|\alpha_{1}\right|=m_{1}}\left(\int_{R^{n}}\left|D^{\alpha_{1}} \tilde{b}_{1}(x) f_{1}(x)\right|^{r} w(x) d x\right)^{1 / r}
\end{array}
$$




$$
\begin{array}{lrl}
\leq & C \sum_{\left|\alpha_{2}\right|=m_{2}}\left\|D^{\alpha_{2}} b_{2}\right\|_{B M O} w(Q)^{-1 / r} \sum_{\left|\alpha_{1}\right|=m_{1}}\left(\int_{\tilde{Q}}\left|D^{\alpha} \tilde{b}_{1}(x)\right|^{r s} d x\right)^{1 / r s} \\
\times & \left(\int_{\tilde{Q}}|f(x)|^{p} w(x) d x\right)^{1 / p}\left(\int_{\tilde{Q}} w(x)^{(1-r / p) t} d x\right)^{1 / r t} \\
\leq & C \prod_{j=1}^{2}\left(\sum_{\left|\alpha_{j}\right|=m_{j}}\left\|D^{\alpha_{j}} b_{j}\right\|_{B M O}\right) w(Q)^{-1 / r}|Q|^{1 / r s}\left\|f \chi_{\tilde{Q}}\right\|_{L^{p}(w)}\left(\frac{1}{|\tilde{Q}|} \int_{\tilde{Q}} w(x) d x\right)^{p_{0} / r t} \\
\leq & C \prod_{j=1}^{2}\left(\sum_{\left|\alpha_{j}\right|=m_{j}}\left\|D^{\alpha_{j}} b_{j}\right\|_{B M O}\right) w(\tilde{Q})^{-1 / p}\left\|f \chi_{\tilde{Q}}\right\|_{L^{p}(w)} \\
\leq & C \prod_{j=1}^{2}\left(\sum_{\left|\alpha_{j}\right|=m_{j}}\left\|D^{\alpha_{j}} b_{j}\right\|_{B M O}\right)\|f\|_{B_{p}(w)}, \\
L_{3} \leq & C \prod_{j=1}^{2}\left(\sum_{\left|\alpha_{j}\right|=m_{j}}\left\|D^{\alpha_{j}} b_{j}\right\|_{B M O}\right)\|f\|_{B_{p}(w) .} .
\end{array}
$$

For $L_{4}$, taking $r, s_{1}, s_{2}, t>1$ such that $r<p, t=p p_{0} /(p-r)$ and $1 / s_{1}+1 / s_{2}+$ $1 /(p / r)+1 / t=1$, then, by the reverse of Hölder's inequality,

$$
\begin{array}{rlrl}
L_{4} & \leq & C \sum_{\left|\alpha_{1}\right|=m_{1},\left|\alpha_{2}\right|=m_{2}}\left(\frac{1}{w(Q)} \int_{R^{n}}\left|T\left(D^{\alpha_{1}} \tilde{b}_{1} D^{\alpha_{2}} \tilde{b}_{2} f_{1}\right)(x)\right|^{r} w(x) d x\right)^{1 / r} \\
& \leq & C w(Q)^{-1 / r} \sum_{\left|\alpha_{1}\right|=m_{1},\left|\alpha_{2}\right|=m_{2}}\left(\int_{R^{n}}\left|D^{\alpha_{1}} \tilde{b}_{1}(x) D^{\alpha_{2}} \tilde{b}_{2}(x) f_{1}(x)\right|^{r} w(x) d x\right)^{1 / r} \\
& \leq & C w(Q)^{-1 / r} \sum_{\left|\alpha_{1}\right|=m_{1},\left|\alpha_{2}\right|=m_{2}}\left(\int_{\tilde{Q}}\left|D^{\alpha} \tilde{b}_{1}(x)\right|^{r s_{1}} d x\right)^{1 / r s_{1}}\left(\int_{\tilde{Q}}\left|D^{\alpha} \tilde{b}_{2}(x)\right|^{r s_{2}} d x\right)^{1 / r s_{2}} \\
& \times & \left(\int_{\tilde{Q}}|f(x)|^{p} w(x) d x\right)^{1 / p}\left(\int_{\tilde{Q}} w(x)^{(1-r / p) t} d x\right)^{1 / r t} \\
& \times & C \prod_{j=1}^{2}\left(\sum_{\left|\alpha_{j}\right|=m_{j}}\left\|D^{\alpha_{j}} b_{j}\right\|_{B M O}\right) w(Q)^{-1 / r}|Q|^{1 / r s_{1}+1 / r s_{2}+1 / r t} \\
& \leq & C \prod_{j=1}^{2}\left(\sum_{\left|\alpha_{j}\right|=m_{j}}\left\|D^{\alpha_{j}} b_{j}\right\|_{B M O}\right) w(\tilde{Q})^{-1 / p}\left\|f \chi_{\tilde{Q}}\right\|_{L^{p}(w)} \\
& C \prod_{j=1}^{2}\left(\sum_{\left|\alpha_{j}\right|=m_{j}}\left\|D^{\alpha_{j}} b_{j}\right\|_{B M O}\right)\|f\|_{B_{p}(w)} .
\end{array}
$$

For $L_{5}$, similar to the proof of the proof of $I_{5}$ in Theorem 1, we have

$$
T_{\tilde{b}}\left(f_{2}\right)(x)-T_{\tilde{b}}\left(f_{2}\right)(0)=\int_{R^{n}}\left(\frac{K(x-y)}{|x-y|^{m}}-\frac{K(-y)}{|y|^{m}}\right) \prod_{j=1}^{2} R_{m_{j}}\left(\tilde{b}_{j} ; x, y\right) f_{2}(y) d y
$$




$$
\begin{aligned}
& +\quad \int_{R^{n}}\left(R_{m_{1}}\left(\tilde{b}_{1} ; x, y\right)-R_{m_{1}}\left(\tilde{b}_{1} ; 0, y\right)\right) \frac{R_{m_{2}}\left(\tilde{b}_{2} ; x, y\right)}{|y|^{m}} K(-y) f_{2}(y) d y \\
& +\quad \int_{R^{n}}\left(R_{m_{2}}\left(\tilde{b}_{2} ; x, y\right)-R_{m_{2}}\left(\tilde{b}_{2} ; 0, y\right)\right) \frac{R_{m_{1}}\left(\tilde{b}_{1} ; x_{0}, y\right)}{|y|^{m}} K(-y) f_{2}(y) d y \\
& -\quad \sum_{\left|\alpha_{1}\right|=m_{1}} \frac{1}{\alpha_{1} !} \int_{R^{n}}\left[\frac{R_{m_{2}}\left(\tilde{b}_{2} ; x, y\right)(x-y)^{\alpha_{1}}}{|x-y|^{m}} K(x-y)-\frac{R_{m_{2}}\left(\tilde{b}_{2} ; 0, y\right)(-y)^{\alpha_{1}}}{|y|^{m}} K(-y)\right] \\
& D^{\alpha_{1}} \tilde{b}_{1}(y) f_{2}(y) d y \\
& -\quad \sum_{\left|\alpha_{2}\right|=m_{2}} \frac{1}{\alpha_{2} !} \int_{R^{n}}\left[\frac{R_{m_{1}}\left(\tilde{b}_{1} ; x, y\right)(x-y)^{\alpha_{2}}}{|x-y|^{m}} K(x-y)-\frac{R_{m_{1}}\left(\tilde{b}_{1} ; 0, y\right)(-y)^{\alpha_{2}}}{|y|^{m}} K(-y)\right] \\
& \times \quad D^{\alpha_{2}} \tilde{b}_{2}(y) f_{2}(y) d y \\
& +\quad \sum_{\left|\alpha_{1}\right|=m_{1},\left|\alpha_{2}\right|=m_{2}} \frac{1}{\alpha_{1} ! \alpha_{2} !} \int_{R^{n}}\left[\frac{(x-y)^{\alpha_{1}+\alpha_{2}}}{|x-y|^{m}} K(x-y)-\frac{(-y)^{\alpha_{1}+\alpha_{2}}}{|y|^{m}} K(-y)\right] \\
& \times \quad D^{\alpha_{1}} \tilde{b}_{1}(y) D^{\alpha_{2}} \tilde{b}_{2}(y) f_{2}(y) d y \\
& =\quad L_{5}^{(1)}(x)+L_{5}^{(2)}(x)+L_{5}^{(3)}(x)+L_{5}^{(4)}(x)+L_{5}^{(5)}(x)+L_{5}^{(6)}(x) .
\end{aligned}
$$

For $L_{5}^{(1)}(x)$, taking $1<q, r<\infty$ such that $1 / p+1 / q+1 / r=1$, by $w \in A_{1} \subset$ $A_{p / r+1}$, we get

$$
\begin{aligned}
& \left|L_{5}^{(1)}(x)\right| \leq \\
& C \sum_{k=0}^{\infty} \int_{2^{k+1} \tilde{Q} \backslash 2^{k} \tilde{Q}}\left|\frac{1}{|x-y|^{m}}-\frac{1}{|y|^{m}}\right| \\
& |K(x-y)| \prod_{j=1}^{2}\left|R_{m_{j}}\left(\tilde{b}_{j} ; x, y\right)\right||f(y)| w(y)^{1 / p} w(y)^{-1 / p} d y \\
& +\quad \sum_{k=0}^{\infty} \int_{2^{k+1} \tilde{Q} \backslash 2^{k} \tilde{Q}}|K(x-y)-K(-y)||y|^{-m} \\
& \times \quad \prod_{j=1}^{2}\left|R_{m_{j}}\left(\tilde{b}_{j} ; x, y\right)\right||f(y)| w(y)^{1 / p} w(y)^{-1 / p} d y \\
& \leq C \prod_{j=1}^{2}\left(\sum_{|\alpha|=m_{j}}\left\|D^{\alpha} b_{j}\right\|_{B M O}\right) \sum_{k=0}^{\infty} k^{2} 2^{-k}\left(\int_{2^{k+1} \tilde{Q} \backslash 2^{k}} \tilde{Q}|K(x-y)|^{q} d y\right)^{1 / q} \\
& \times \quad\left(\int_{2^{k+1} \tilde{Q}}|f(y)|^{p} w(y) d y\right)^{1 / p}\left(\int_{2^{k+1} \tilde{Q}} w(y)^{-r / p} d y\right)^{1 / r} \\
& +\quad C \prod_{j=1}^{2}\left(\sum_{|\alpha|=m_{j}}\left\|D^{\alpha} b_{j}\right\|_{B M O}\right) \\
& \times \quad \sum_{k=0}^{\infty} k^{2}\left(\int_{2^{k+1} \tilde{Q} \backslash 2^{k} \tilde{Q}}|K(x-y)-K(-y)|^{q} d y\right)^{1 / q}
\end{aligned}
$$




$$
\begin{array}{cc}
\times & \left(\int_{2^{k+1} \tilde{Q}}|f(y)|^{p} w(y) d y\right)^{1 / p}\left(\int_{2^{k+1} \tilde{Q}} w(y)^{-r / p} d y\right)^{1 / r} \\
\leq & C \prod_{j=1}^{2}\left(\sum_{\left|\alpha_{j}\right|=m_{j}}\left\|D^{\alpha_{j}} b_{j}\right\|_{B M O}\right) \\
\times & \sum_{k=1}^{\infty} k^{2} 2^{-k} w\left(2^{k} \tilde{Q}\right)^{-1 / p}\left(\int_{2^{k}}|f(y)|^{p} w(y) d y\right)^{1 / p} \\
\times & \left(\frac{1}{\left|2^{k} \tilde{Q}\right|} \int_{2^{k}} \tilde{Q} w(y) d y\right)^{1 / p}\left(\frac{1}{\left|2^{k} \tilde{Q}\right|} \int_{2^{k} \tilde{Q}} w(y)^{-1 /(p-1)} d y\right)^{(p-1) / p} \\
+ & \quad C \prod_{j=1}^{2}\left(\sum_{\left|\alpha_{j}\right|=m_{j}}\left\|D^{\alpha_{j}} b_{j}\right\|_{B M O}\right) \\
\times & \sum_{k=1}^{\infty} k^{2} 2^{-k a} w\left(2^{k} \tilde{Q}\right)^{-1 / p}\left(\int_{2^{k}}|f(y)|^{p} w(y) d y\right)^{1 / p} \\
\times & \left(\frac{1}{\left|2^{k} \tilde{Q}\right|} \int_{2^{k} \tilde{Q}} w(y) d y\right)^{1 / p}\left(\frac{1}{\left|2^{k} \tilde{Q}\right|} \int_{2^{k}} \tilde{Q} w(y)^{-r / p} d y\right)^{1 / r} \\
\leq & C \prod_{j=1}^{2}\left(\sum_{\left|\alpha_{j}\right|=m_{j}}\left\|D^{\alpha_{j}} b_{j}\right\|_{B M O}\right)\|f\|_{B_{p}(w)} .
\end{array}
$$

Similarly, we get, for $1<q, r_{1}, r_{2}, r_{3}, s<\infty$ with $1 / p+1 / q+1 / r_{1}+1 / s=1$ and $1 / p+1 / q+1 / r_{2}+1 / r_{3}+1 / s=1$,

$$
\begin{aligned}
& \left|L_{5}^{(2)}(x)+L_{5}^{(3)}(x)+L_{5}^{(4)}(x)+L_{5}^{(5)}(x)+L_{5}^{(6)}(x)\right| \\
& \leq \quad C\left(\sum_{|\alpha|=m_{2}}\left\|D^{\alpha} b_{2}\right\|_{B M O}\right) \sum_{|\alpha|=m_{1}} \sum_{k=0}^{\infty} \int_{2^{k+1} \tilde{Q} \backslash 2^{k} \tilde{Q}} \frac{k d}{2^{k} d}\left|K(-y)\left\|D^{\alpha_{1}} \tilde{b}_{1}(y)\right\| f(y)\right| d y \\
& +\quad C\left(\sum_{|\alpha|=m_{1}}\left\|D^{\alpha} b_{1}\right\|_{B M O}\right) \sum_{|\alpha|=m_{2}} \sum_{k=0}^{\infty} \int_{2^{k+1}} \tilde{Q} \backslash 2^{k} \tilde{Q} \frac{k d}{2^{k} d}\left|K(-y)\left\|D^{\alpha_{2}} \tilde{b}_{2}(y)\right\| f(y)\right| d y \\
& +\quad C\left(\sum_{|\alpha|=m_{2}}\left\|D^{\alpha} b_{2}\right\|_{B M O}\right) \sum_{|\alpha|=m_{1}} \sum_{k=0}^{\infty} k \int_{2^{k+1} \tilde{Q} \backslash 2^{k} \tilde{Q}}\left|K(x-y)-K(-y) \| D^{\alpha_{1}} \tilde{b}_{1}(y)\right||f(y)| d y \\
& +\quad C\left(\sum_{|\alpha|=m_{1}}\left\|D^{\alpha} b_{1}\right\|_{B M O}\right) \sum_{|\alpha|=m_{2}} \sum_{k=0}^{\infty} k \int_{2^{k+1} \tilde{Q} \backslash 2^{k} \tilde{Q}}\left|K(x-y)-K(-y)\left\|D^{\alpha_{2}} \tilde{b}_{2}(y)\right\| f(y)\right| d y \\
& +\quad C \sum_{\left|\alpha_{1}\right|=m_{1},\left|\alpha_{2}\right|=m_{2}} \sum_{k=0}^{\infty} \int_{2^{k+1} \tilde{Q} \backslash 2^{k} \tilde{Q}}\left|K(x-y)-K(-y)\left\|D^{\alpha_{1}} \tilde{b}_{1}(y)\right\| D^{\alpha_{2}} \tilde{b}_{2}(y) \| f(y)\right| d y \\
& \leq \quad C\left(\sum_{|\alpha|=m_{2}}\left\|D^{\alpha} b_{2}\right\|_{B M O}\right) \sum_{|\alpha|=m_{1}} \sum_{k=0}^{\infty} k 2^{-k}\left(\int_{2^{k+1} \underline{\tilde{Q}}}\left|D^{\alpha_{1}} \tilde{b}_{1}(y)\right|^{r_{1}} d y\right)^{1 / r_{1}} \\
& \times \quad\left(\int_{2^{k+1} \tilde{Q} \backslash 2^{k} \tilde{Q}}|K(-y)|^{q} d y\right)^{1 / q}\left(\int_{2^{k+1} \tilde{Q}}|f(y)|^{p} w(y) d y\right)^{1 / p}\left(\int_{2^{k+1} \tilde{Q}} w(y)^{-s / p} d y\right)^{1 / s}
\end{aligned}
$$




$$
\begin{aligned}
& +\quad C\left(\sum_{|\alpha|=m_{1}}\left\|D^{\alpha} b_{1}\right\|_{B M O}\right) \sum_{|\alpha|=m_{2}} \sum_{k=0}^{\infty} k 2^{-k}\left(\int_{2^{k+1} \underline{\tilde{Q}}}\left|D^{\alpha_{2}} \tilde{b}_{2}(y)\right|^{r_{1}} d y\right)^{1 / r_{1}} \\
& \times \quad\left(\int_{2^{k+1} \tilde{Q} \backslash 2^{k} \tilde{Q}}|K(-y)|^{q} d y\right)^{1 / q}\left(\int_{2^{k+1} \tilde{Q}}|f(y)|^{p} w(y) d y\right)^{1 / p}\left(\int_{2^{k+1} \tilde{Q}} w(y)^{-s / p} d y\right)^{1 / s} \\
& +\quad C\left(\sum_{|\alpha|=m_{2}}\left\|D^{\alpha} b_{2}\right\|_{B M O}\right) \sum_{|\alpha|=m_{1}} \sum_{k=0}^{\infty} k\left(\int_{2^{k+1} \tilde{Q} \backslash 2^{k} \tilde{Q}}|K(x, y)-K(0, y)|^{q} d y\right)^{1 / q} \\
& \times \quad\left(\int_{2^{k+1} \tilde{Q}}\left|D^{\alpha_{1}} \tilde{b}_{1}(y)\right|^{r_{1}} d y\right)^{1 / r_{1}}\left(\int_{2^{k+1} \tilde{Q}}|f(y)|^{p} w(y) d y\right)^{1 / p}\left(\int_{2^{k+1} \tilde{Q}} w(y)^{-s / p} d y\right)^{1 / s} \\
& +\quad C\left(\sum_{|\alpha|=m_{1}}\left\|D^{\alpha} b_{1}\right\|_{B M O}\right) \sum_{|\alpha|=m_{2}} \sum_{k=0}^{\infty} k\left(\int_{2^{k+1} \tilde{Q} \backslash 2^{k} \tilde{Q}}|K(x, y)-K(0, y)|^{q} d y\right)^{1 / q} \\
& \times \quad\left(\int_{2^{k+1} \tilde{Q}}\left|D^{\alpha_{2}} \tilde{b}_{2}(y)\right|^{r_{1}} d y\right)^{1 / r_{1}}\left(\int_{2^{k+1} \tilde{Q}}|f(y)|^{p} w(y) d y\right)^{1 / p}\left(\int_{2^{k+1} \tilde{Q}} w(y)^{-s / p} d y\right)^{1 / s} \\
& +\quad C \sum_{\left|\alpha_{1}\right|=m_{1},\left|\alpha_{2}\right|=m_{2}} \sum_{k=0}^{\infty}\left(\int_{2^{k+1} \tilde{Q} \backslash 2^{k} \tilde{Q}}|K(x, y)-K(0, y)|^{q} d y\right)^{1 / q}\left(\int_{2^{k+1} \tilde{Q}} w(y)^{-s / p} d y\right)^{1 / s} \\
& \times \quad\left(\int_{2^{k+1} \tilde{Q}}\left|D^{\alpha_{1}} \tilde{b}_{1}(y)\right|^{r_{2}} d y\right)^{1 / r_{2}}\left(\int_{2^{k+1} \tilde{Q}}\left|D^{\alpha_{2}} \tilde{b}_{2}(y)\right|^{r_{3}} d y\right)^{1 / r_{3}}\left(\int_{2^{k+1} \tilde{Q}}|f(y)|^{p} w(y) d y\right)^{1 / p} \\
& \leq C \prod_{j=1}^{2}\left(\sum_{\left|\alpha_{j}\right|=m_{j}}\left\|D^{\alpha_{j}} b_{j}\right\|_{B M O}\right) \sum_{k=1}^{\infty} k^{2}\left(2^{-k}+2^{-k a}\right) w\left(2^{k} \tilde{Q}\right)^{-1 / p}\left(\int_{2^{k} \tilde{Q}}|f(y)|^{p} w(y) d y\right)^{1 / p} \\
& \leq \quad C \prod_{j=1}^{2}\left(\sum_{\left|\alpha_{j}\right|=m_{j}}\left\|D^{\alpha_{j}} b_{j}\right\|_{B M O}\right)\|f\|_{B_{p}(w)} .
\end{aligned}
$$

Thus

$$
L_{5} \leq C \prod_{j=1}^{2}\left(\sum_{\alpha_{j} \mid=m_{j}}\left\|D^{\alpha_{j}} b_{j}\right\|_{B M O}\right)\|f\|_{B_{p}(w)} .
$$

This finishes the proof of Theorem 2.

\section{ACKNOWLEDGEMENT}

Project supported by the Science and Technology Department of Hunnan Province Science and Technology Plan Projects(2011fj3130)

\section{REFERENCES}

[1] J. Cohen, "A sharp estimate for a multilinear singular integral on $R^{n}$," Indiana Univ. Math. J., vol. 30, no. 1, pp. 693-702, 1985, doi: 10.1512/iumj.1981.30.30053.

[2] J. Cohen and J. Gosselin, “On multilinear singular integral operators on $R^{n}$," Studia Math., vol. 72, no. 2, pp. 199-223, 1982.

[3] J. Cohen and J. Gosselin, "A BMO estimate for multilinear singular integral operators," Illinois J. Math., vol. 30, no. 3, pp. 445-465, 1986. 
[4] R. Coifman and Y. Meyer, Wavelets, Calderón-Zygmund and multilinear operators, 1st ed., ser. Cambridge Studies in Advanced Mathematics. Cambridge: Cambridge University Press, 1997, vol. 48 .

[5] R. R. Coifman, R. and G. Weiss, "Factorization theorems for Hardy spaces in several variables," Ann. of Math., vol. 103, no. 5, pp. 611-635, 1976, doi: 10.2307/1970954.

[6] J. Garcia-Cuerva and J. L. Rubio de Francia, Weighted norm inequalities and related topics, 1st ed., ser. North-Holland Math. Amsterdam: North-Holland, 1985, vol. 116.

[7] E. Harboure, C. Segovia, and J. L. Torrea, "Boundedness of commutators of fractional and singular integrals for the extreme values of p," Illinois J. Math., vol. 41, no. 5, pp. 676-700, 1997.

[8] D. S. Kurtz and R. L. Wheeden, "Results on weighted norm inequalities for multiplies," Trans. Amer. Math. Soc., vol. 225, no. 2, pp. 343-362, 1979, doi: 10.1090/S0002-9947-1979-0542885-8.

[9] L. Z. Liu, "Weighted boundedness of multilinear operators for the extreme," Taiwanese J. of Math., vol. 10, no. 5, pp. 669-690, 2006.

[10] L. Z. Liu, "Weighted Herz type spaces estimates of multilinear singular integral operators for the extreme cases," Revista de la Real Academia de Ciencias Exactas, Serie A: Mate., vol. 101, no. 5 , pp. 587-598, 2007.

[11] L. Z. Liu, "Endpoint estimates for multilinear fractional singular integral operators on some Hardy spaces," Math. Notes, vol. 88, no. 6, pp. 735-752, 2010.

[12] S. Z. Lu and D. C. Yang, "Multiplier theorem for Herz type Hardy spaces," Proc. Amer. Math. Soc., vol. 126, no. 22, pp. 3337-3346, 1998, doi: 10.1090/S0002-9939-98-04931-4.

[13] C. Pérez and G. Pradolini, "Sharp weighted endpoint estimates for commutators of singular integral operators," Michigan Math. J., vol. 49, no. 1, pp. 23-37, 2001, doi: 10.1307/mmj/1008719033.

[14] C. Pérez and R. Trujillo-Gonzalez, "Sharp weighted estimates for multilinear commutators," J. London Math. Soc., vol. 65, no. 5, pp. 672-692, 2002, doi: 10.1112/S0024610702003174

[15] E. M. Stein, Harmonic analysis: real variable methods, orthogonality and oscillatory integrals, 1st ed., ser. 43. Princeton: Princeton, 1993, vol. 43.

[16] K. W. Wang and L. Z. Liu, "Sharp inequality for multilinear commutator of multiplier operator," Acta Math. Vietnamica, vol. 34, no. 2, pp. 233-244, 2009.

[17] Z. You, "Results of commutators obtained norm inequalities," Adv. in Math.(in Chinese), vol. 17, no. 1 , pp. $79-84,1988$.

[18] P. Zhang and J. C. Chen, "The $\left(L^{p}, \dot{F}_{p}^{\beta, \infty}\right)$-boundedness of commutators of multiplies," Acta Math. Sinica, vol. 21, no. 4, pp. 765-772, 2005.

[19] P. Zhang and J. C. Chen, "Boundedness properties for commutators of multiplies," Acta Math. Sinica(Chinese Series), vol. 49, no. 6, pp. 1387-1396, 2006.

Authors' addresses

Jinsong Pan

Hunan Mechanical and Electrical Polytechnic, Changsha 410151, P. R. of China

E-mail address: pjs196855@126.com

\section{Lijuan Tong}

Hunan Mechanical and Electrical Polytechnic, Changsha 410151, P. R. of China 